\title{
Two modified Zagreb indices for random structures
}

https://doi.org/10.1515/mgmc-2021-0013

received August 01, 2020; accepted March 02, 2021

Abstract: Random structure plays an important role in the composition of compounds, and topological index is an important index to measure indirectly the properties of compounds. The Zagreb indices and its revised versions (or redefined versions) are frequently used chemical topological indices, which provide the theoretical basis for the determination of various physical-chemical properties of compounds. This article uses the tricks of probability theory to determine the reduced second Zagreb index and hyper-Zagreb index of two kinds of vital random graphs: $G(n, p)$ and $G(n, m)$.

Keywords: theoretical chemistry, Zagreb index, random graph, hyper-Zagreb index, second Stirling number

\section{Introduction}

The Zagreb index named as the capital of Croatia is one of the first chemical topological indices to be defined. The research on Zagreb index has a long history. It has always been the primary chemical topological index studied by theoretical chemists and has a wide range of applications in various chemical engineering fields. The contributions in Zagreb indices in recent years can be referred to Ali et al. (2020), Ashrafi et al. (2019), Aslam et al. (2019), Buyantogtokh et al. (2020), Došlić et al. (2020), Du et al. (2019), Furtula et al. (2019), Gao et al. (2018b, 2019, 2020), Javaid et al. (2019), Noureen et al. (2020), Siddiqui (2020), and Wang et al. (2020). All the random graphs considered

* Corresponding author: Siman Li, Department of Biochemistry and Molecular Biology, Kunming Medical University, Kunming 650500, China, e-mail: kmlisiman@163.com

Li Shi: Institute of Medical Biology, Chinese Academy of Medical Sciences and Peking Union Medical College, Kunming 650092, China Wei Gao: School of Information Science and Technology, Yunnan Normal University, Kunming 650500, China in our article are simple graphs, that is, loops, multi-edges and directed edges are not considered here.

The traditional first and second Zagreb indices are defined by:

$$
M_{1}(G)=\sum_{v \in V(G)}(d(v))^{2}
$$

and

$$
M_{2}(G)=\sum_{u v \in E(G)} d(u) d(v),
$$

respectively. In recent ten years, various of variants of Zagreb index are introduced in different engineering applications. For instance, modified second Zagreb index is formulated by:

$$
M_{2}^{\star}(G)=\sum_{u v \in E(G)} \frac{1}{d(u) d(v)} .
$$

Furthermore, different version and revised forms of Zagreb index are defined one after another. For example, first multiplicative Zagreb index and second multiplicative Zagreb index are stated as follows:

$$
\begin{aligned}
& \Pi_{1}^{\star}(G)=\prod_{v \in V(G)}(d(v))^{2}, \\
& \Pi_{2}^{\star}(G)=\prod_{u v \in E(G)} d(u) d(v),
\end{aligned}
$$

In this paper, we focus on the following two versions of Zagreb index:

- $\quad$ Reduced second Zagreb index:

$$
R M_{2}(G)=\sum_{u v \in E(G)}(d(u)-1)(d(v)-1) .
$$

- Hyper-Zagreb index:

$$
H M(G)=\sum_{e=u v \in E(G)}(d(u)+d(v))^{2} .
$$


The research on random graphs has penetrated into every field of science, even social sciences. The most famous example is "six degrees of separation", which is simply stated that the diameter of social random network graph does not exceed 6. In recent years, random graphs and random chemical structures have received extensive attention and been fully studied (see: Dommers et al., 2018; Gao et al., 2017, 2018a; Klein et al., 2004; Kouri et al., 2015; Limkumnerd, 2014; Škrekovski et al., 2019; and Tang et al., 2017).

There are two kinds of noted random graphs:

- $G(n, p):|V(G(n, p))|=n$, and whether there is an edge between two vertices determined by $p \in(0,1)$;

- $\quad G(n, m):|V(G(n, m))|=n$ and $|E(G(n, m))|=m$.

Random graphs $G(n, p)$ and $G(n, m)$ have an important research position in random networks and chemical structures. In the "six degrees of separation" social network, people are known each other by a certain probability. For example, a teaching staff working at Kunming Medical University (in short, KMU), and his academic ability neither too strong nor too poor. If too strong, he will work in a top university. If too poor, he can' meet the requirements of KMU. Similarly, students' college entrance examination scores must not be too high, otherwise they will enter a first-class university. Also not be too low, otherwise they will not meet the admission requirements of KMU. Among the universities where students' scores allow to be entered, KMU was chosen so that the teaching staff and his students know each other. Therefore, $p$ in $G(n, p)$ is used to describe the probability of two people meeting each other. In chemistry, it describes the random connection between atoms constitutes the form of molecular structure.

Let $A=\left[a_{i j}\right] \in\{0,1\}^{n \times n}$ be adjacency matrix of graph $G$ with $n$ vertices where $a_{i j}=1$ if and only if $v_{i} v_{j} \in E(G)$ and otherwise $a_{i j}=0$. It is clear thateachrandom graphin $G(n, p)$ yields a random symmetric $(0,1)$-matrix in which each entry above the main diagonal with probability $p$ is equal to one, and vice versa. Moreover, every random graph in $G(n, m)$ obtains a random symmetric $(0,1)$-matrix which contains exactly $m$ entries 1 above the main diagonal, and vice versa. In this way, the investigation of random graphs $G(n, p)$ and $G(n, m)$ can be transformed to the research of corresponding random symmetric $(0,1)$-matrices.

The main contribution of this paper is to study the reduced second Zagreb index and hyper-Zagreb index of $G(n, p)$ and $G(n, m)$. The rest sections are organised as follows: we first introduce some prepare knowledge which will be used in the third section; then, the main conclusions and detailed proofs are given; finally, we discuss the future ongoing works.

\section{Prerequisite knowledge}

The main purpose in this section is to list the degree distribution polynomial, Stirling number and indicator random variables which will be used in the proofing of main results.

Let $\delta(G)$ and $\Delta(G)$ be the minimum and maximum degree of graph $G$. The degree sequence polynomial $S_{G}(x)$ with degree sequence $\delta(G)=d_{1} \leq \cdots \leq d_{n}=\Delta(G)$ is defined as generating polynomial by Sedghi et al. (2008) which is formulated by:

$$
S_{G}(x)=\sum_{v \in V(G)} x^{d(v)}=\sum_{i=\delta(G)}^{\Delta(G)} a_{i} x^{i},
$$

where $a_{i}=|\{v \in V(G): d(v)=i\}|$. By simple computations, we acquire $S_{G}(1)=|V(G)|$ and $S_{G}^{\prime}(1)=2|E(G)|$.

Let $D_{v}$ be a random variable corresponding to the degree of vertex $v \in V(G)$ where $G \in G(n, p)$. Then its vertex degree distribution can be denoted as:

$$
\mathrm{P}\left(D_{v}=t\right)=\left(\begin{array}{c}
n-1 \\
t
\end{array}\right) p^{t}(1-p)^{n-1-t} .
$$

Došlić et al. (2020) introduced the corresponding polynomial function which is stated by:

$$
f_{n, p}(x)=\sum_{t=0}^{n-1}\left(\begin{array}{c}
n-1 \\
t
\end{array}\right) p^{t}(1-p)^{n-1-t} x^{t}=(1-p+p x)^{n-1} .
$$

In terms of directly calculating, we infer that for any $i \in\{1, \cdots, n-1\}$,

$$
f_{n, p}^{(i)}(1)=(n-1) \cdots(n-i) p^{i} .
$$

In combinatorial theory, it is well-known that there are two main types of Stirling numbers where the first kind of Stirling number (here denoted it by $\left.\left[\begin{array}{l}n \\ t\end{array}\right]\right)$ expresses the number of ways to arrange $n$ objects into cycles, and the second kind of Stirling number (here denoted it by $\left\{\begin{array}{l}n_{t} \\ t\end{array}\right\}$ ) expresses the number of partitions of a set with $n$ elements into $t$ non-empty subsets. It satisfies the following recursion condition:

$$
\left\{\begin{array}{l}
n \\
t
\end{array}\right\}=t\left\{\begin{array}{c}
n-1 \\
t
\end{array}\right\}+\left\{\begin{array}{l}
n-1 \\
t-1
\end{array}\right\}
$$

where $n \in N$ and the initial conditions are $\left\{\begin{array}{l}0 \\ 0\end{array}\right\}=1$ and $\left\{\begin{array}{l}0 \\ i\end{array}\right\}=\left\{\begin{array}{l}j \\ 0\end{array}\right\}=0$ for any $i, j>0$. Let $x^{\underline{t}}=x(x-1) \cdots(x-t+1)$ be falling factorial. We deduce:

$$
x^{\underline{n}}=\sum_{t}\left[\begin{array}{l}
n \\
t
\end{array}\right](-1)^{n-t} x^{t}
$$




$$
x^{n}=\sum_{t}\left\{\begin{array}{l}
n \\
t
\end{array}\right\} x^{\underline{t}}
$$

More details on the characters and applications of Stirling number can be referred to Arratia and DeSalvo (2017), Bagno et al. (2019), Ballantine and Merca (2018), Benyi et al. (2019), Kuba and Panholzer (2019), Maltenfort (2020), Mansour and Shattuck (2018), Merca (2019), and Prodinger (2019).

Let $\mathbf{1}=\left(\begin{array}{c}1 \\ \vdots \\ 1\end{array}\right)$ be all 1 vector and $D=A \mathbf{1}$ ( $A$ is adjacency matrix of random graph $G$, i.e., a random symmetric $(0,1)$ matrix). For a random graph $G$ with $V(G)=\left\{v_{1}, \cdots, v_{n}\right\}$ the indicator random variables $X_{i j}$ for $i, j \in\{1, \cdots, n\}$ is formulated by:

$$
X_{i j}=\left\{\begin{array}{l}
1, \text { if } v_{v} v_{j} \in E(G) \\
0, \text { otherwise }
\end{array} .\right.
$$

Obviously, $X_{i i}=0$ and $X_{i j}=X_{j i}$. We consider the following two situations:

- $G \in G(n, p): \quad X_{i j}$ and $X_{r s}$ are independent for $i, j, r, s \in\{1, \cdots, n\}$ and $\{i, j\} \neq\{\stackrel{r s}{\neq}\{r, s\}$;

- $G \in G(n, m): X_{i j}$ and $X_{r s}$ are not independent for $i, j, r, s \in\{1, \cdots, n\}$.

Let $\mathrm{E}(\cdot)$ be expectation function. It is well-known that $\mathrm{E}(X Y)=\mathrm{E}(X) \mathrm{E}(Y)$ if $X$ and $Y$ are independent random varioables. For $G(n, m)$ case and $i \in\{1,2,3\}$, set (defined by Došlić et al., 2020)

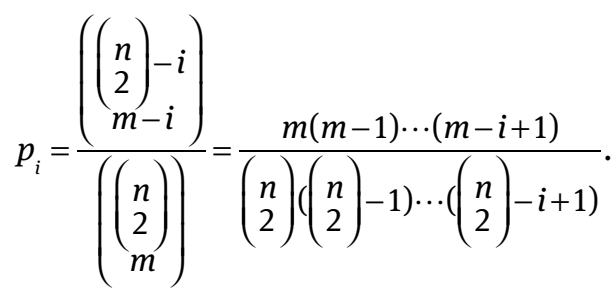

\section{Results and proofs}

The main theorem for random graph $G(n, p)$ is presented as follows.

\section{Theroem 1}

Let $G \in G(n, p)$. Then:

$$
\mathrm{E}\left(R M_{2}(G)\right)=\left(\begin{array}{l}
n \\
2
\end{array}\right)(n-2)^{2} p^{3},
$$

$$
\begin{aligned}
\mathrm{E}(H M(G))= & 2 n(n-1) p+5 n(n-1)(n-2) p^{2} \\
& +n(n-1)(n-2)(2 n-5) p^{3} .
\end{aligned}
$$

Proof. Set $V(G)=\left\{v_{1}, \cdots, v_{n}\right\}$ and $D_{i}$ as the corresponding random variable of vertex $v_{i}$ for $i \in\{1, \cdots, n\}$. We get:

$$
\mathrm{E}\left(R M_{2}(G)\right)=\mathrm{E}\left(\sum_{u v \in E(G)}(d(u)-1)(d(v)-1)\right)
$$$$
=\mathrm{E}\left(\sum_{u v \in E(G)}(d(u) d(v)-d(u)-d(v)+1)\right)
$$$$
=\mathrm{E}\left(\sum_{u v \in E(G)}(d(u) d(v))\right)-\mathrm{E}\left(\sum_{u v E(G)}(d(u)+d(v))\right)+\mathrm{E}(|E(G)|)
$$$$
=\mathrm{E}\left(\sum_{v_{i} v_{j} \in E(G)}\left(d\left(v_{i}\right) d\left(v_{j}\right)\right)\right)-\mathrm{E}\left(\sum_{v \in V(G)} d^{2}(v)\right)+\left(\begin{array}{l}
n \\
2
\end{array}\right) p
$$$$
=\mathrm{E}\left(\sum_{v_{i} v_{j} \in E(G)} D_{i} D_{j}\right)-\mathrm{E}\left(\sum_{v \in V(G)} D_{v}^{2}\right)+\left(\begin{array}{l}
n \\
2
\end{array}\right) p
$$$$
=\frac{1}{2} \mathrm{E}\left(\sum_{i=1}^{n} \sum_{j=1, i \neq j}^{n} D_{i} D_{j} X_{i j}\right)-\sum_{v \in V(G)} \mathrm{E}\left(D_{v}^{2}\right)+\left(\begin{array}{l}
n \\
2
\end{array}\right) p
$$$$
=\frac{1}{2} \sum_{i=1}^{n} \sum_{j=1, i \neq j}^{n} \mathrm{E}\left(D_{i} D_{j} X_{i j}\right)-\sum_{v \in V(G)} \sum_{t=0}^{n-1} t^{2}\left(\begin{array}{c}
n-1 \\
t
\end{array}\right) p^{t}(1-p)^{n-1-t}
$$$$
+\left(\begin{array}{l}
n \\
2
\end{array}\right) p
$$

$$
\begin{aligned}
& =\frac{1}{2} \sum_{i=1}^{n} \sum_{j=1, i \neq j}^{n} \sum_{k=1, k \neq i}^{n} \sum_{r=1, r \neq j}^{n} \mathrm{E}\left(X_{i k} X_{j r} X_{i j}\right) \\
& -\sum_{v \in V(G)} \sum_{t=0}^{n-1} \sum_{j=1}^{2}\left\{\begin{array}{l}
2 \\
j
\end{array}\right\} t^{j}\left(\begin{array}{c}
n-1 \\
t
\end{array}\right) p^{t}(1-p)^{n-1-t}+\left(\begin{array}{l}
n \\
2
\end{array}\right) p \\
& =\frac{1}{2} \sum_{i=1}^{n} \sum_{j=1, i \neq j}^{n}\left\{\mathrm{E}\left(X_{i j} X_{j i} X_{i j}\right)+\sum_{k=1, k \neq i, j}^{n} \mathrm{E}\left(X_{i k}\right) \mathrm{E}\left(X_{j i} X_{i j}\right)\right. \\
& +\sum_{r=1, r \neq j, i}^{n} \mathrm{E}\left(X_{j r}\right) \mathrm{E}\left(X_{i j} X_{i j}\right) \\
& \left.+\sum_{k=1, k \neq i, j}^{n} \sum_{r=1, r \neq j, i}^{n} \mathrm{E}\left(X_{i k}\right) \mathrm{E}\left(X_{j r}\right) \mathrm{E}\left(X_{i j}\right)\right\} \\
& -\sum_{v \in V(G)} \sum_{j=1}^{2}\left\{\begin{array}{l}
2 \\
j
\end{array}\right\} \sum_{t=0}^{n-1} t^{j}\left(\begin{array}{c}
n-1 \\
t
\end{array}\right) p^{t}(1-p)^{n-1-t}+\left(\begin{array}{l}
n \\
2
\end{array}\right) p
\end{aligned}
$$




$$
\begin{array}{ll}
=\frac{1}{2} \sum_{i=1}^{n} \sum_{j=1, i \neq j}^{n}\left(p+2(n-2) p^{2}+(n-2)^{2} p^{3}\right) & \left.+\sum_{r=1, r \neq j, i}^{n} \mathrm{E}\left(X_{j r}\right) \mathrm{E}\left(X_{i j} X_{i j}\right)+\sum_{k=1, k \neq i, j r=1, r \neq j, i}^{n} \sum_{i k}^{n} \mathrm{E}\left(X_{i k}\right) \mathrm{E}\left(X_{j r}\right) \mathrm{E}\left(X_{i j}\right)\right\} \\
-n \sum_{j=1}^{2}\left\{\begin{array}{l}
2 \\
j
\end{array}\right\} \frac{n !}{(n-j-1) !} p^{j}+\left(\begin{array}{l}
n \\
2
\end{array}\right) p & =\sum_{v \in V(G)} \sum_{j=1}^{3}\left\{\begin{array}{l}
3 \\
j
\end{array}\right\} \sum_{t=0}^{n-1} t^{i}\left(\begin{array}{c}
n-1 \\
t
\end{array}\right) p^{t}(1-p)^{n-1-t} \\
=\frac{n^{2}-n}{2}\left(p+2(n-2) p^{2}+(n-2)^{2} p^{3}\right) & +\sum_{i=1}^{n} \sum_{j=1, i \neq j}^{n}\left(p+2(n-2) p^{2}+(n-2)^{2} p^{3}\right) \\
-n(n-1) p((n-2) p+1)+\left(\begin{array}{l}
n \\
2
\end{array}\right) p & =n \sum_{j=1}^{3}\left\{\begin{array}{l}
3 \\
j
\end{array}\right\} \frac{n !}{(n-j-1) !} p^{j}+\left(n^{2}-n\right)\left(p+2(n-2) p^{2}\right. \\
=\left(\begin{array}{l}
n \\
2
\end{array}\right)\left(2 p+2(n-2) p^{2}+(n-2)^{2} p^{3}\right) & \left.+(n-2)^{2} p^{3}\right) \\
-\left(\begin{array}{l}
n \\
2
\end{array}\right)\left(2(n-2) p^{2}+2 p\right) & =n(n-1) p+3 n(n-1)(n-2) p^{2}+n(n-1)(n-2)(n-3) p^{3} \\
=\left(\begin{array}{l}
n \\
2
\end{array}\right)(n-2)^{2} p^{3} . & +\left(n(n-1) p+2 n(n-1)(n-2) p^{2}\right. \\
\mathrm{E}(H M(G))=\mathrm{E}\left(\sum_{e=u v \in E(G)}(d(u)+d(v))^{2}\right) & \left.+n(n-1)(n-2)^{2} p^{3}\right) \\
& =2 n(n-1) p+5 n(n-1)(n-2) p^{2} \\
& +n(n-1)(n-2)(2 n-5) p^{3} .
\end{array}
$$$$
=\mathrm{E}\left(\sum_{u v \in E(G)}\left(d^{2}(u)+d^{2}(v)+2 d(u) d(v)\right)\right)
$$$$
=\mathrm{E} \sum_{u v \in[(G)}\left(d^{2}(u)+d^{2}(v)\right)+2 \mathrm{E} \sum_{u v \in(G)}(d(u) d(v))
$$$$
=\mathrm{E}\left(\sum_{v \in V(G)} d^{3}(v)\right)+2 \mathrm{E}\left(\sum_{v_{i} v_{j} \in E(G)} D_{i} D_{j}\right)
$$$$
=\mathrm{E}\left(\sum_{v \in V(G)} D_{v}^{3}\right)+\mathrm{E}\left(\sum_{i=1}^{n} \sum_{j=1, i \neq j}^{n} D_{i} D_{j} X_{i j}\right)
$$$$
=\sum_{v \in V(G)} \mathrm{E}\left(D_{v}^{3}\right)+\sum_{i=1}^{n} \sum_{j=1, i \neq j}^{n} \mathrm{E}\left(D_{i} D_{j} X_{i j}\right)
$$$$
=\sum_{v \in V(G)} \sum_{t=0}^{n-1} t^{3}\left(\begin{array}{c}
n-1 \\
t
\end{array}\right) p^{t}(1-p)^{n-1-t}
$$$$
+\sum_{i=1}^{n} \sum_{j=1, i \neq j}^{n} \sum_{k=1, k \neq i}^{n} \sum_{r=1, r \neq j}^{n} \mathrm{E}\left(X_{i k} X_{j r} X_{i j}\right)
$$$$
=\sum_{v \in V(G)} \sum_{t=0}^{n-1} \sum_{j=1}^{3}\left\{\begin{array}{l}
3 \\
j
\end{array}\right\} t^{\underline{j}}\left(\begin{array}{c}
n-1 \\
t
\end{array}\right) p^{t}(1-p)^{n-1-t}
$$$$
+\sum_{i=1}^{n} \sum_{j=1, i \neq j}^{n}\left\{\mathrm{E}\left(X_{i j} X_{j i} X_{i j}\right)+\sum_{k=1, k \neq i, j}^{n} \mathrm{E}\left(X_{i k}\right) \mathrm{E}\left(X_{i j} X_{i j}\right)\right.
$$

Thus, we get the desired conclusions. The second main theorem for random graph $G(n, m)$ is manifested as follows.

\section{Theroem 2}

Let $G \in G(n, m)$. Then:

$$
\begin{aligned}
& \mathrm{E}\left(R M_{2}(G)\right)=-\frac{n^{2}-n}{2} p_{1}+\frac{n^{2}-n}{2}(n-2)^{2} p_{3}+m, \\
& \mathrm{E}(H M(G))=2 n(n-1) p_{1}+5 n(n-1)(n-2) p_{2} \\
& \quad+n(n-1)(n-2)(2 n-5) p_{3} .
\end{aligned}
$$

Proof. Set $V(G)=\left\{v_{1}, \cdots, v_{n}\right\}$ and $D_{i}$ as the corresponding random variable of vertex $v_{i}$ for $i \in\{1, \cdots, n\}$. We get:

$$
\begin{aligned}
& \mathrm{E}\left(R M_{2}(G)\right)=\mathrm{E}\left(\sum_{u v \in E(G)}(d(u)-1)(d(v)-1)\right) \\
& =\mathrm{E}\left(\sum_{u v \in E(G)}(d(u) d(v)-d(u)-d(v)+1)\right) \\
& =\mathrm{E}\left(\sum_{u v E E(G)}(d(u) d(v))\right)-\mathrm{E}\left(\sum_{u v \in E(G)}(d(u)+d(v))\right)+\mathrm{E}(|E(G)|) \\
& =\mathrm{E}\left(\sum_{v_{i} v_{j} \in E(G)}\left(d\left(v_{i}\right) d\left(v_{j}\right)\right)\right)-\mathrm{E}\left(\sum_{v \in V(G)} d^{2}(v)\right)+m
\end{aligned}
$$




$$
\begin{aligned}
& =\mathrm{E}\left(\sum_{v_{i} v_{j} \in E(G)} D_{i} D_{j}\right)-\mathrm{E}\left(\sum_{v \in V(G)} D_{v}^{2}\right)+m \\
& =\frac{1}{2} \mathrm{E}\left(\sum_{i=1}^{n} \sum_{j=1, i \neq j}^{n} D_{i} D_{j} X_{i j}\right)-\sum_{v \in V(G)} \mathrm{E}\left(D_{v}^{2}\right)+m \\
& =\frac{1}{2} \sum_{i=1}^{n} \sum_{j=1, i \neq j}^{n} \mathrm{E}\left(D_{i} D_{j} X_{i j}\right)-\sum_{i=1}^{n} E\left(\left(\sum_{k=1, k \neq i}^{n} X_{i k}\right)^{2}\right)+m \\
& =\frac{1}{2} \sum_{i=1}^{n} \sum_{j=1, i \neq j}^{n} \sum_{k=1, k \neq i}^{n} \sum_{r=1, r \neq j}^{n} \mathrm{E}\left(X_{i k} X_{j r} X_{i j}\right)-\left(\sum_{i=1}^{n} \mathrm{E}\left(\sum_{k=1, k \neq i}^{n} X_{i k}^{2}\right)\right. \\
& +\sum_{i=1}^{n} \mathrm{E}\left(\sum_{k=1, k \neq i}^{n} \sum_{r=1, r \neq i, k}^{n}\left(X_{i k} X_{i r}\right)\right)+m \\
& =\frac{1}{2} \sum_{i=1}^{n} \sum_{j=1, i \neq j}^{n}\left\{\mathrm{E}\left(X_{i j} X_{j i} X_{i j}\right)+\sum_{k=1, k \neq i, j}^{n} \mathrm{E}\left(X_{i k} X_{j i} X_{i j}\right)\right. \\
& +\sum_{r=1, r \neq j, i}^{n} \mathrm{E}\left(X_{j r} X_{i j} X_{i j}\right) \\
& \left.+\sum_{k=1, k \neq i, j}^{n} \sum_{r=1, r \neq j, i}^{n} \mathrm{E}\left(X_{i k} X_{j r} X_{i j}\right)\right\}-\left(\sum_{i=1}^{n} \sum_{k=1, k \neq i}^{n} \mathrm{E}\left(X_{i k}^{2}\right)\right. \\
& \left.+\sum_{i=1}^{n} \sum_{k=1, k \neq i}^{n} \sum_{r=1, r \neq i, k}^{n} \mathrm{E}\left(X_{i k} X_{i r}\right)\right)+m \\
& =\frac{1}{2} \sum_{i=1}^{n} \sum_{j=1, i \neq j}^{n}\left(p_{1}+2(n-2) p_{2}+(n-2)^{2} p_{3}\right)-\left(n(n-1) p_{1}\right. \\
& \left.+n(n-1)(n-2) p_{2}\right)+m \\
& =\frac{n^{2}-n}{2}\left(p_{1}+2(n-2) p_{2}+(n-2)^{2} p_{3}\right) \\
& -\left(n(n-1) p_{1}+n(n-1)(n-2) p_{2}\right)+m \\
& =-\frac{n^{2}-n}{2} p_{1}+\frac{n^{2}-n}{2}(n-2)^{2} p_{3}+m \\
& \mathrm{E}(H M(G))=\mathrm{E}\left(\sum_{e=u v \in E(G)}(d(u)+d(v))^{2}\right) \\
& =\mathrm{E}\left(\sum_{u v \in E(G)}\left(d^{2}(u)+d^{2}(v)+2 d(u) d(v)\right)\right) \\
& =\mathrm{E} \sum_{u v \in E(G)}\left(d^{2}(u)+d^{2}(v)\right)+2 \mathrm{E} \sum_{u v \in E(G)}(d(u) d(v))
\end{aligned}
$$

$$
\begin{aligned}
& =\mathrm{E}\left(\sum_{v \in V(G)} d^{3}(v)\right)+2 \mathrm{E}\left(\sum_{v_{i} v_{j} \in E(G)} D_{i} D_{j}\right) \\
& =\mathrm{E}\left(\sum_{v \in V(G)} D_{v}^{3}\right)+\mathrm{E}\left(\sum_{i=1}^{n} \sum_{j=1, i \neq j}^{n} D_{i} D_{j} X_{i j}\right) \\
& =\sum_{v \in V(G)} \mathrm{E}\left(D_{v}^{3}\right)+\sum_{i=1}^{n} \sum_{j=1, i \neq j}^{n} \mathrm{E}\left(D_{i} D_{j} X_{i j}\right) \\
& =\sum_{i=1}^{n} E\left(\left(\sum_{k=1, k \neq i}^{n} X_{i k}\right)^{3}\right)+\sum_{i=1}^{n} \sum_{j=1, i \neq j}^{n} \sum_{k=1, k \neq i}^{n} \sum_{r=1, r \neq j}^{n} \mathrm{E}\left(X_{i k} X_{j r} X_{i j}\right) \\
& =\left\{\sum_{i=1}^{n} \mathrm{E}\left(\sum_{k=1, k \neq i}^{n} X_{i k}^{3}\right)+3 \sum_{i=1}^{n} \sum_{k=1, k \neq i}^{n} \mathrm{E}\left(X_{i k}^{2}\left(\sum_{r=1, r \neq i, k}^{n} X_{i r}\right)\right)\right. \\
& \left.+\sum_{i=1}^{n} \mathrm{E}\left(\sum_{k=1, k \neq i}^{n} \sum_{r=1, r \neq i, k}^{n} \sum_{s=1, s \neq i, k, \mathrm{r}}^{n} X_{i k} X_{i r} X_{i s}\right)\right\} \\
& +\sum_{i=1}^{n} \sum_{j=1, i \neq j}^{n}\left\{\mathrm{E}\left(X_{i j} X_{j i} X_{i j}\right)+\sum_{k=1, k \neq i, j}^{n} \mathrm{E}\left(X_{i k} X_{j i} X_{i j}\right)\right. \\
& +\sum_{r=1, r \neq j, i}^{n} \mathrm{E}\left(X_{j r} X_{i j} X_{i j}\right) \\
& \left.+\sum_{k=1, k \neq i, j}^{n} \sum_{r=1, r \neq j, i}^{n} \mathrm{E}\left(X_{i k} X_{j r} X_{i j}\right)\right\} \\
& =\left\{\sum_{i=1}^{n} \sum_{k=1, k \neq i}^{n} \mathrm{E}\left(X_{i k}^{3}\right)+3 \sum_{i=1}^{n} \sum_{k=1, k \neq i}^{n} \sum_{r=1, r \neq i, k}^{n} \mathrm{E}\left(X_{i k}^{2} X_{i r}\right)\right. \\
& \left.+\sum_{i=1}^{n} \sum_{k=1, k \neq i}^{n} \sum_{r=1, r \neq i, k}^{n} \sum_{s=1, s \neq i, k, \mathrm{r}}^{n} \mathrm{E}\left(X_{i k} X_{i r} X_{i s}\right)\right\} \\
& +\sum_{i=1}^{n} \sum_{j=1, i \neq j}^{n}\left(p_{1}+2(n-2) p_{2}+(n-2)^{2} p_{3}\right) \\
& =n(n-1) p_{1}+3 n(n-1)(n-2) p_{2}+n(n-1)(n-2)(n-3) p_{3} \\
& +n(n-1)\left(p_{1}+2(n-2) p_{2}+(n-2)^{2} p_{3}\right) \\
& =2 n(n-1) p_{1}+5 n(n-1)(n-2) p_{2} \\
& +n(n-1)(n-2)(2 n-5) p_{3} \text {. }
\end{aligned}
$$

Therefore, we complete the proof of Theorem 2 .

Theorem 1 and Theorem 2 presented the means of reduced second Zagreb index and hyper-Zagreb index of $G(n, p)$ and $G(n, m)$ presectively, which reflect the central location of indices of random graphs and the central tendency of random index values. 


\section{Conclusion and discussion}

Random structure plays an important role in the synthesis of chemical molecular structure, and the analysis of random graph helps us understand the characteristics of compound molecular structure under probabilistic conditions. In this paper, we determine the reduced second Zagreb index and hyper-Zagreb index of $G(n, p)$ and $G(n, m)$ by means of probability tricks and mathematical derivation.

The following topics can be considered as the future ongoing works:

- More topological chemcial indices of random graphs $G(n, p)$ and $G(n, m)$ should be discussed;

- More kinds of random graphs should be considered as well;

- It is stated in Newman et al. (2001) that the vertex degree distribution function has been in different expressions of different settings. Hence, the special topological chemical indices for specific random graphs (i.e., Poisson-distributed graphs, exponentially distributed graphs, and power-law distributed graphs) can be studied in the future.

Acknowledgements: Authors thank the editors and reviewers for their constructive comments in improving the quality of this paper.

Funding information: This research was funded by the Outstanding Youth Science Fundation of Yunnan Basic Research Project [202001AW070021], the Reserve Talent Project for Young and Middle-aged Acadamic and Technical Leaders [2020005AC160023], and Innovative Research Team of Yunnan Province [202005AE160002].

Author contributions: Siman Li: writing - original draft, methodology, project administration; Li Shi: formal analysis; Wei Gao: writing - review and editing.

Conflict of interest: Authors state no conflict of interest.

Data availability statement: Authors declare that this paper has been developed without any data.

\section{References}

Ali U., Javaid M., Kashif A., Modified Zagreb connection indices of the T-sum graphs. Main Group Met. Chem., 2020, 43(1), 43-55.
Arratia R., DeSalvo S., Completely effective error bounds for Stirling numbers of the first and second kinds via poisson approximation. Ann. Comb., 2017, 21(1), 1-24.

Ashrafi A.R., Eliasi M., Ghalavand A., Laplacian coefficients and Zagreb indices of trees. Linear Multilinear A., 2019, 67(9), 1736-1749.

Aslam A., Nadeem M.F., Zahid Z., Zafar S., Gao W., Computing certain topological indices of the line graphs of subdivision graphs of some rooted product graphs. Mathematics, 2019, 7(5), 393, DOI: $10.3390 /$ math7050393.

Bagno E., Biagioli R., Garber D., Some identities involving second kind Stirling numbers of types B and D. Electron. J. Combin., 2019, 26(3), UNSP P3.9.

Ballantine C., Merca M., Euler-Riemann Zeta function and ChebyshevStirling numbers of the first kind. Mediterr. J. Math., 2018, 15(3), DOI: 10.1007/s00009-018-1172-2.

Benyi B., Mendez M., Ramirez J.L., Wakhare T., Restricted $r$-Stirling numbers and their combinatorial applications. Appl. Math. Comput., 2019, 348, 186-205.

Buyantogtokh L., Horoldagva B., Das K.C., On reduced second Zagreb index. J. Comb. Optim., 2020, 39(3), 776-791.

Dommers S., Giardina C., Giberti C., van der Hofstad R., Large deviations for the annealed ising model on inhomogeneous random graphs: spins and degrees. J. Stat. Phys., 2018, 173(3-4), 1045-1081.

Došlić T., Hosseinzadeh M.A., Hossein-Zadeh S., Iranmanesh A., Rezakhanlou F., On generalized Zagreb indices of random graphs. MATCH-Commun. Math. Co., 2020, 84(2), 499-511.

Du Z.B., Ali A., Trinajstic N., Alkanes with the first three maximal/ minimal modified first Zagreb connection indices. Mol. Inform., 2019, 38(4), DOI: 10.1002/minf.201800116.

Furtula B., Gutman I., Matejic M., Milovanovic E., Milovanovic I., Some new lower bounds for augmented Zagreb index. J. Appl. Math. Comput., 2019, 61(1-2), 405-415.

Gao W., Baig A.Q., Khalid W., Farahani M.R., Molecular description of copper(II) oxide. Maced. J. Chem. Chem. Eng., 2017, 36(1), 93-99.

Gao W., Guirao J.L.G., Abdel-Aty M., Xi W.F., An independent set degree condition for fractional critical deleted graphs. Discrete Cont. Dyn. S., 2019, 12(4-5), 877-886.

Gao W., Guirao J.L.G., Wu H.L., Nordhaus-Gaddum type inequalities for some distance-based indices of bipartite molecular graphs. J. Math. Chem., 2020, 58(7), 1345-1352.

Gao W., Wang W.F., Dimitrov D., Wang Y.Q., Nano properties analysis via fourth multiplicative $A B C$ indicator calculating. Arab. J. Chem., 2018a, 11(6), 793-801.

Gao W., Wu H.L., Siddiqui M.K., Baig A.Q., Study of biological networks using graph theory. Saudi J. Bio. Sci., 2018b, 25(6), 1212-1219.

Javaid F., Jamil M.K., Tomescu I., Extremal k-generalized quasi unicyclic graphs with respect to first and second Zagreb indices. Discrete Appl. Math., 2019, 270, 153-158.

Klein D.J., Palacios J.L., Randic M., Trinajstic N., Random walks and chemical graph theory. J. Chem. Inf. Comp. Sci., 2004, 44(5), 1521-1525.

Kouri T.M., Pascua D., Mehta D.P., Random models and analyses for chemical graphs. Internat. J. Found. Comput. Sci., 2015, 26(2), 269-291. 
Kuba M., Panholzer A., A note on harmonic number identities, Stirling series and multiple zeta values. Int. J. Number Theory, 2019, 15(7): 1323-1348.

Limkumnerd S., Random walk on lattices: graph-theoretic approach to simulating long-range diffusion-attachment growth models. Phys. Rev. E. 2014, 89(3), DOI: 10.1103/PhysRevE.89.032402.

Maltenfort M., New definitions of the generalized Stirling numbers. Aequationes Math., 2020, 94(1), 169-200.

Mansour T., Shattuck M., A generalized class of restricted Stirling and Lah numbers. Math. Slovaca, 2018, 68(4), 727-740.

Merca M., Some notes on the $(q, t)$-Stirling numbers. Discrete Math., 2019, 342(3), 628-634.

Newman M.E.J., Strogatz S.H., Watts D.J., Random graphs with arbitrary degree distributions and their applications. Phys. Rev. E, 2001, 64, 026118.

Noureen S., Bhatti A.A., Ali A., Extremal trees for the modified first Zagreb connection index with fixed number of segments or vertices of degree 2. J. Taibah Univ. Sci., 2020, 14(1), 31-37.
Prodinger H., Logarithms of a binomial series: a Stirling number approach. Ars Math. Contemp., 2019, 17(1): 271-275.

Sedghi S., Shobe N., Salahshoor M. A., The polynomials of a graph. Iran. J. Math. Sci. Inf., 2008, 3, 55-68.

Siddiqui H.M.A., Computation of Zagreb indices and Zagreb polynomials of Sierpinski graphs. Hacet. J. Math. Stat., 2020, 49(2), 754-765.

Škrekovski R., Dimitrov D., Zhong J.M., Wu H.L., Gao W., Remarks on multiplicative atom-bond connectivity index. IEEE Access, 2019, 7(1), 76806-76811.

Tang M., Athreya A., Sussman D.L., Lyzinski V., Park Y., Priebe C.E., A semiparametric two-sample hypothesis testing problem for random graphs. J. Comput. Graph. Statist., 2017, 26(2), 344-354.

Wang Z., Chaudhry F., Naseem M., Asghar A., Reverse Zagreb and reverse hyper-Zagreb indices for crystallographic structure of molecules. J. Chem.-NY, 2020, DOI: 10.1155/2020/ 9805829 . 